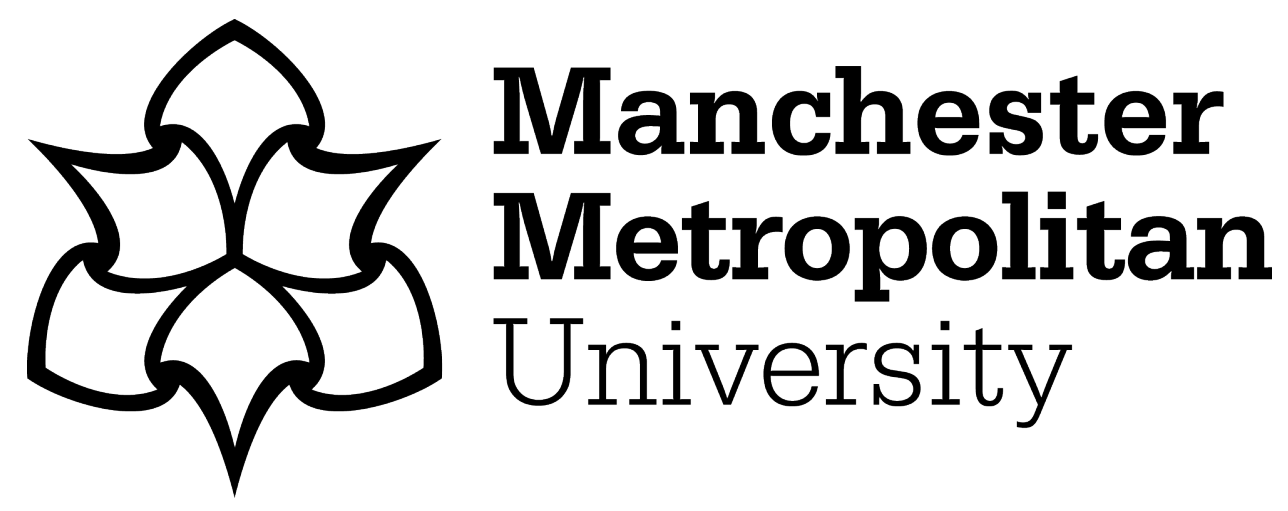

Liu, Y, Rowe, M, Holderbaum, William and Potter, B (2016) A novel battery network modelling using constraint differential evolution algorithm optimisation. Knowledge-Based Systems, 99. pp. 10-18. ISSN 0950-7051

Downloaded from: https://e-space.mmu.ac.uk/620866/

Publisher: Elsevier

DOI: https://doi.org/10.1016/j.knosys.2016.01.019

Usage rights: Creative Commons: Attribution-Noncommercial-No Derivative Works 4.0

Please cite the published version 


\title{
A Novel Battery Network Modelling Using Constraint Differential Evolution Algorithm Optimisation
}

\author{
Yang Liu ${ }^{1}$, Matthew Rowe ${ }^{1}$, William Holderbaum ${ }^{1}$, Ben Potter ${ }^{1}$ \\ Energy Research Lab, \\ School of System Engineering, Reading University, Reading, RG6 6AY, UK
}

\begin{abstract}
The amount of battery storage into the power system network has been increasing in the recent years. The use of battery storage devices has been advocated as one of the main ways of improving the power quality and reliability of the power system, including minimization of energy imbalance and reduction of peak demand. Higher peaks in demand will increase the electricity price and could cause blackouts and infrastructure damage. Lowering peak demand to reduce the use of carbon-intensive fuels and the number of expensive peaking plant generators is thus of major importance. Self-adaptive control methods for individual batteries have been developed to reduce the peak demand. However, these self-adaptive control algorithms of are not very efficient without sharing the energy among different batteries. This paper proposes a novel battery network system with optimal management of energy between batteries. An optimal management strategy has been implemented using a population-based constraint differential evolution algorithm. Taking advantage of this strategy the battery network model can remove more peak areas of forecasted demand data compared to the self-adaptive control algorithm developed for the New York City study case.
\end{abstract}

Keywords: Optimisation; Constraint Optimisation; Self-adaptive Control; Distributed Network Model 


\section{Introduction}

Battery energy storage has found a wide range of applications in various fields of science. Batteries can improve the power quality (mainly voltage depressions and power interruptions) and reliability of power system (Divya and $\emptyset$ stergaard, 2009). Battery storage could also play a vital role in deferring the need to improve the transmission and distribution capacity to meet ever growing power demand by effectively increasing the capacity of a given network by reducing peaks. In recent years, the capital cost of battery storage technologies has significantly reduced, thus justifying a new study of its applications (Mercier et al. 2009). For example, some of earliest commercial use of battery storage device were at Bewag, Germany (17MW/14MWh battery for frequency regulation) and at Southern California Edison Chino substation (10MW/40MWh for load leveling, rapid spinning reserve and instantaneous frequency control) (Daly, 1995; Taylor, 1996). The earliest transportable battery (lead-acid), located at Phoenix distribution system is a multi-model battery (Tahllam and Eckroad, 1999). The battery switches between improving power quality (2MW up to $15 \mathrm{~s}$ ) and improving power management (200KW for $45 \mathrm{~min}$ ) and uses a different mode for each model. The megawatt scale deployment of the Distributed Energy Storage system (DES) technology was also successfully carried out in the American electricity power grid in 2006 (Nguyen and Flueck, 2012; Nourai and Schafer, 2009). Higher peaks in demand will increase the electricity price and could cause blackouts and infrastructure damage. Lowering peak demand to reduce the use of carbon-intensive fuels and reduce the number of expensive peaking plant generators is thus of major importance. The Charleston storage project partially funded by the U.S Department of Energy (DOE) aimed to reduce the peak load on overloaded equipment in the distribution substation (Nourai and Schafer, 2009). It has operated successfully for three winter and summer peak seasons. Realizing multiple 
benefits that DES technology has to offer, the utility continued to install three 2-MW, 14.4-MWh NaS DES units of larger capacity in their distribution system in 2008 providing peak shaving. The key feature of the new system is triggered peak shaving that does not allow the battery to be discharged unnecessarily during daily peak hours and only discharges the battery when the load of a nearby "bottleneck" on the grid exceeds a certain "trigger". This approach not only allows the battery to offer its peak shaving value but also increase the availability of the remaining storage energy to serve customers in the event of an outage. Despite the large number of investigations carried out to apply different storage technologies to power system, very few of them have been implemented in practice. One of the main reasons for this limited practical application is lack of practical experience and lack of availability of tools which could be used for optimal control of battery storage in the smart grid during planning. Lately there has been some development of different types of optimal control algorithms in smart grid (Zhou et al., 2010; Coppez et al., 2010; Vytelingum et al., 2010).

Coppez et al. (2010) has classified battery storage optimisations based on hybrid renewable energy system in four categories: graph construction, probabilistic and deterministic techniques, genetic algorithms and artificial neural networks. Main issues like cell battery technology and optimisation techniques were reviewed. The authors stated that the reliability of supply of the system must be kept in mind to ensure that the load will be met by the supply at all times and economically the system must be optimized to ensure the lowest cost possible whist maintaining the system integrity. A common parameter used to measure the system integrity and reliability is Loss of Power Supply Probability (LPSP). LPSP must be monitored as the key parameter to ensure that in optimizing the system, the likelihood of the system supply not being able to meet the load at all times is kept very low. Graphical construction is used to optimize in terms of two 
criteria (either Photovoltaic (PV) and size of battery storage, or PV and wind turbine) (Borowy and Salameh, 1996). However, some important factors (such as the PV module slope angle and the wind turbine installation height.) were completely neglected. Other techniques will prove more useful for a more complex system with high dimensional parameters because it is only useful for simple systems with few parameters. Probabilistic techniques can be used in situations where actual hour by hour long-term data is not available and more general data needs to be used (Coppez et al., 2010). The probabilistic and deterministic techniques are achieved by initially creating a design space of feasible solutions which adhere to the maximum LPSP. The parameters such as the number of wind turbines, size of PV panels and size of battery storage are optimized using the objective function (e.g. cost of the system including PV modules, Batteries, wind turbines and the cost of design and installation). Tina et al. (2006) presented a probabilistic approach based on the convolution technique to incorporate the fluctuating nature of the resources and load, thus eliminating the need for time-series data, to assess the long-term performance of a hybrid solar wind system for both-stand-alone and grid-connected applications. Performance of the hybrid system under study is assessed by employing probabilistic models for both PV array and wind turbines. Disadvantage of this probabilistic approach is that it cannot represent the dynamic changing performance of the hybrid system (Zhou et al., 2010). Ould Bila et al. (2010) show a case study of the optimisation of a wind, PV and battery distributed generation system in Senegal. A genetic algorithm (GA) was used to minimize the total cost of the system whist maintaining a low LPSP using the following parameters: number of PV modules, power output of wind turbines, battery capacity and number of inverters and regulators (Ould Bila et al., 2010). The system is now functioning optimally. GAs were selected because they have shown to be highly applicable to cases of non-linear systems, where the location of the 
global optimum is a difficult task (Zhou et al., 2010). A Neural Networks (NNs) was used to predict the fitness values of solutions in order to speed up the GA search process (Zhou et al., 2008). This approach substantially decreases the time taken to calculate the optimal solution, while keeping the accuracy of each of the methods. The system includes the photovoltaic arrays, the lead-acid battery and a flywheel. The optimal sizing can be considered as a constrained optimization problem: minimization the total capacity of energy storage system, subject to the main constraint of the Loss of Power Supply Probability (LPSP) (Zhou et al., 2008). The GA spent 45 minutes but the combinatorial optimization by GA and NNs) spent only 3-5 minutes on calculation.

In addition, Vytelingum et al. (2010) developed a novel agent-based micro-storage management of energy storage devices in UK homes that adapts to market condition using game theory optimisation. They show that using demand-side management (i.e., directly controlling the storage profile of a number of homes) coupled with storage can increase savings made in the system. In the UK electricity market, it is possible to achieve savings of up to $13 \%$ on average for a consumer on his electricity bill with a storage device of $4 \mathrm{kWh}$. In spite of benefits in using the advanced agent-based model for the smart grid, the cost of micro-storage devices for all UK homes makes it impractical to apply the proposed method and the optimal control of storage details haven't been given in the paper. A self-adaptive control model (SACM) of individual battery storage was developed by Rowe et al. (2012) to remove the peaks of forecasted demand. The SACM was applied to Bracknell, UK using individual battery. However, the self-adaptive control algorithm of individual battery is not very efficient to reduce peaks without sharing the energy among different batteries. This paper proposes a novel Battery Network Model (BNM) with optimal management between batteries in the network. Mathematically, the optimal 
management of battery network is a large scale constraint optimisation with the objective of maximally removing the peak areas of forecasted demand or actual demand.

The optimisation methods can be broadly divided into two groups: linear and nonlinear optimisation methods. Linear optimisation's characteristics are a linear objective function to be maximized (or minimized) and linear constraints (i.e. constraints are linear functions of the variables). For some nonlinear optimisation problems, due to non-convexity, the objective function may have many local optima, and an analytical expression of the objective function may not be available. Nonlinear optimisation methods may be classified into deterministic local optimisation methods (e.g., gradient methods or direct search methods) and stochastic global optimisation methods (examples are multiple local search, genetic algorithms, simulated annealing and tabu search) (Andersson, 2000; Holland, 1975; Press et al., 1992; Cvijovic, 1995). Stochastic optimisation refers to the minimisation (or maximisation) of a function in the presence of randomness in the optimisation process. Genetic algorithms (GAs) (Holland, 1975) and particle swam optimisation (PSO) (Kennedy and Eberhart, 1995) and differential evolution (DE) (Storn and Price, 1995) are popular stochastic optimisations for better global optimisation frameworks to fully realize the full benefits to conducting mathematical model optimisation, because of their simplicity, global perspective, and inherent parallel processing (Deb, 2000; Liu, 2009; Liu and Pender, 2012 ).

In most cases of practical interest, global optimisation is very difficult. This is because of the omnipresence of local minimum, the number of which tends to increase exponentially with the size of the problem (Cvijovic, 1995). Conventional minimisation techniques, which are time consuming and tend to converge to whichever local minimum they first encounter in such cases. The solution in these cases may not be the global minimum but a local minimum sensitive to the 
starting point. Also these methods are unable to continue the search after a local minimum is reached. Mathematical models may have many local optima on the objective function surface, and in such cases local search is inappropriate because the estimated optimum will depend on the starting point of the search. Due to the high number of possible parameter combinations, computation becomes very expensive for complex models if using a method based on searching combinations of parameters (Shen, 2006). The particle swarm optimisation and differential evolution are two efficient stochastic optimisation methods minimizing an objective function that can model the problem's objectives while incorporating constraints, and have three main advantages: global search regardless of the initial parameter values, fast convergence and a few control parameters. Both techniques have shown great promise in several real-world applications (Deb, 2001; Liu \& Khu, 2007; Liu, 2009; Liu \& Sun, 2011; Liu and Pender, 2012; Liu and Pender, 2013). Facts have proved that population based optimisations like GA, PSO and DE are suitable to handle complicated constrained optimisation problems (Coello Coello, 2002; Wang and Cai, 2012). Differential Evolution (DE) is used in the paper to optimize the high-dimensional battery network model parameters because of its robust search ability based on benchmark test functions and real applications among these algorithms (Storn and Price, 1997; Deb, et al., 2010; Vesterstroem and Thomsen, 2004; Liu and Sun, 2010).

The rest of this paper structured as follows. Section 2 and 3 formulate a self-adaptive control approach for an individual battery and our proposed battery network approach. Section 4 describes the constraint differential evolution algorithm for the battery network optimisation. Section 5 empirically studies this system for New York City peak demand reduction through simulation and optimisation. Finally, Section 5 presents conclusions on the work presented in this paper. 


\section{Self-Adaptive Control Model (SACM) of Individual Battery}

Rowe et al. (2012) presented a mathematical model that describes peak areas of actual demand or forecasted demand can be partially removed using an adaptive storage algorithm. The battery storage at $\Delta t$ and minimization function of peaks can be formulated as:

$$
\begin{gathered}
B\left(\Delta t_{i+1}\right)=B\left(\Delta t_{i}\right) \pm Z\left(\Delta t_{i}\right) \\
\text { If } B\left(\Delta t_{i}\right)+Z\left(\Delta t_{i}\right)>B_{c} \quad \text { then } B\left(\Delta t_{i}\right)=B_{c} \quad i=1,2, \ldots, T \\
\text { If } B\left(\Delta t_{i}\right)-Z\left(\Delta t_{i}\right)<0 \text { then } B\left(\Delta t_{i}\right)=0 \\
P_{r}=\operatorname{Minimize}\left(\frac{\sum_{i=1}^{L}\left(Z\left(\Delta t_{i}\right)-B^{*}\left(\Delta t_{i}\right)\right)}{\sum_{i=1}^{N} Z\left(\Delta t_{i}\right)}\right) \text { (Index } i \text { is for peak areas only.) } \\
Z\left(\Delta t_{i}\right)=\int_{t}^{t+\Delta t_{i}} \text { power }(t) d t
\end{gathered}
$$

Where $B\left(\Delta t_{i}\right)$ is energy storage in the battery (kwh) at $\Delta t_{i}, \operatorname{power}(t)$ is the power value (kw) in Figure $1, B_{c}$ is the capacity of the low battery, $L$ is the number of peaks, $Z\left(\Delta t_{i}\right)$ is the charged or discharged area of the actual or forecasted demand curve in Figure 1 and $B^{*}\left(\Delta t_{i}\right)$ is the energy supply to remove the peak demand from battery storage at $\Delta t_{i} . B^{*}\left(\Delta t_{i}\right)$ is decided after optimisation process. Figure 1 shows the forecasted or actual demand with five charged and discharged zones. The horizontal dash line indicates the actual supply threshold from generation or safety limit for the electrical facility. The threshold value depends on capacity of generation and the physical constraints of the network. The self-adaptive control algorithm of individual battery is not very efficient to reduce peaks without sharing the energy among different batteries. Thus, this paper proposes a novel battery network system with optimal management. 


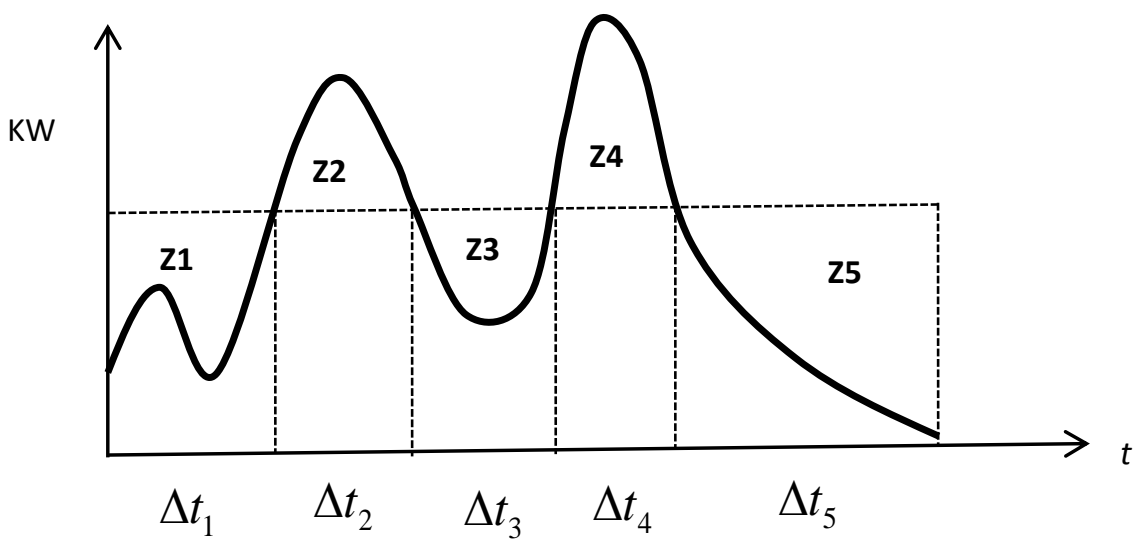

Figure 1: battery storage algorithm

\section{Our Proposed Battery Network Model Algorithm}

The novel battery network modeling approach can be formulated based on the energy conservation law:

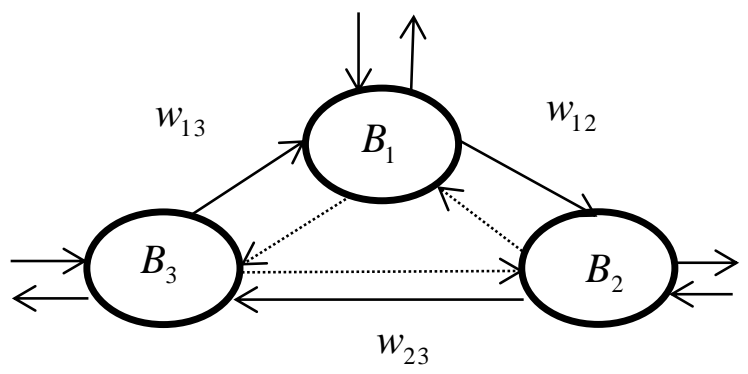

Figure 2: battery network model

$$
\begin{gathered}
B_{1}\left(\Delta t_{l+1}\right)=B_{1}\left(\Delta t_{l}\right)+w_{13}\left(\Delta t_{l}\right)-w_{12}\left(\Delta t_{l}\right) \\
B_{2}\left(\Delta t_{l+1}\right)=B_{2}\left(\Delta t_{l}\right)-w_{23}\left(\Delta t_{l}\right)+w_{12}\left(\Delta t_{l}\right) \\
B_{3}\left(\Delta t_{l+1}\right)=B_{3}\left(\Delta t_{l}\right)+w_{23}\left(\Delta t_{l}\right)-w_{13}\left(\Delta t_{l}\right) \\
0 \leq B_{n}\left(\Delta t_{l}\right) \pm \sum_{j=1}^{M} w_{n j} \leq B_{n c} \quad l=1 \ldots Q
\end{gathered}
$$

where $M$ is the number of the connected nodes, $w_{n j}$ is the battery node $B_{n}$ to borrow energy from its neighbor $B_{j}$ or lend energy to its neighbor $B_{j}$, and the dashed line indicates the weight 
that can be positive or negative. The $Q$ is the total number of sampled data, and the index $l$ is different from index $i$ in formula (6) and formula (1).

$$
P_{r}=\operatorname{Minimize}\left(\sum_{n=1}^{N} \frac{\sum_{i=1}^{L}\left(Z_{n}\left(\Delta t_{i}\right)-B^{*}\left(\Delta t_{i}\right)\right)}{\sum_{i=1}^{L} Z\left(\Delta t_{n i}\right)}\right) \quad(\text { Index } i \text { is for peak areas only; } N=3)
$$

Where $n$ is the number of nodes and $L$ is the total number of peaks. The above is equivalent to minimizing the following formula because all the batteries should keep positive or zero during sharing the electricity:

If $B_{1}\left(\Delta t_{l}\right)<0$ or $B_{2}\left(\Delta t_{l}\right)<0$ or $B_{3}\left(\Delta t_{l}\right)<0$ then

$$
B M=\operatorname{Minimize}\left(\sum_{l=1}^{Q}\left|B_{1}\left(\Delta t_{l}\right)\right|\right)+\operatorname{Min}\left(\sum_{l=1}^{Q}\left|B_{2}\left(\Delta t_{l}\right)\right|\right)+\operatorname{Min}\left(\sum_{l=1}^{Q}\left|B_{3}\left(\Delta t_{l}\right)\right|\right)
$$

If $B_{n}\left(\Delta t_{l}\right)>0$ then $B_{n}\left(\Delta t_{l}\right)=0 \quad n=1 \ldots .3$ in the above formula (11).

The formula shows that battery network algorithm can be easily extended to large scale networks because the same structure can be used with more batteries. Storage system models have been proposed to study the time scale-over a short time scale (minutes and / or seconds) and long time scale (days and / or hours) ( Bejan et al.,2012 ). We will simulate the battery network model around 1 day period for the case study in section 5 .

\section{Constraint Differential Evolution Algorithm}

It is very difficult to converge towards to global optimum for high dimensional optimisation. This is not only because it is constraint problem, but also the curse of dimensionality may increase the convergence time of traditional optimisation methods (e.g. local optimisations) (Jin, 2005). The robust constraint DE search algorithm in this paper is used to optimize the batteries network model parameters. The Constrained DE consists of two major components: the search 
algorithm and the constrained-handling technique. Thus, the performance of constrained DE is primarily dependent on these two components (Wang and Cai, 2012). The aim of the search algorithm is to adjust the exploration and exploitation abilities of population, while the constraint-handling technique focuses how to incorporate the constraints into the DE evolutionary process. DE is a population-based direct-search algorithm for global optimisation (Storn and Price, 1997) which utilizes $P$ D-dimensional parameter vectors. The standard DE works as follows: for each vector $x_{i, G}, i=1,2, \ldots, P$, a trail vector $v$ is generated according to

$$
v_{i, G+1}=x_{r_{1}, G}+F\left(x_{r_{2}, G}-x_{r_{3}, G}\right),
$$

with $r_{1}, r_{2}, r_{3} \in[1, P], P$ is the Population size, $G$ is the iteration index, integer and mutually different, $F>0$, and $r_{1} \neq r_{2} \neq r_{3} \neq i . F$ is a real and constant factor that controls the amplification of the differential variation $\left(x_{r_{2}, G}-x_{r_{3}, G}\right)$. In order to increase the diversity of the parameter vectors, the following vector is adopted:

$$
u_{i, G+1}=\left(u_{1 i, G+1}, u_{2 i, G+1}, \ldots, u_{D i, G+1}\right)
$$

With:

$$
u_{j i, G+1}=\left\{\begin{array}{l}
v_{j i, G+1}, \text { if }(\operatorname{rand}(0,1) \leq C R) \text { or } j=\operatorname{rnbr}(i) \\
x_{j i, G}, \quad \text { if }(\operatorname{rand}(0,1)>C R) \text { and } j \neq \operatorname{rnbr}(i)
\end{array} \quad j=1,2, \ldots, D\right.
$$

Where $D$ is the problem dimension and CR is a user-defined crossover rate. The DE's control variables $(P, F$ and $C R)$ are not difficult to choose in order to obtain good results (Storn and Price, 1997). $F$ and $C R$ are both generally in the range $[0.5,1.0] . \quad r n b r(i)$ is a randomly chosen index from $[1, \mathrm{D}]$. In order to decide whether the new vector $u$ shall become a population member at generation $G+1$, it is compared to $x_{i, G}$. If vector $u$ yields a smaller objective function 
value than $x_{i, G}, x_{i, G+1}$ is set to $u$, otherwise the old value $x_{i, G}$ is retained. The reasonable choice for $P$ between $5 \times \mathrm{D}$ and $10 \times \mathrm{D}$ has been suggested to have enough mutually different vectors with which to work (Storn and Price, 1997). The interesting empirical finding is that rising $P$ does not substantially improve the convergence, independent of the number of parameters for some test cases. This is frequently occurred in real-world optimisation problems rather than artificial test functions (Storn and Price, 1997). As for $F, F=0.5$ is usually a good initial choice. If the population converges prematurely, then $F$ and or $P$ should be increased. $\mathrm{CR}=0.9$ or $\mathrm{CR}=1.0$ is appropriate to first try since a large $\mathrm{CR}$ often speeds convergence.

In most applications, the penalty function method is used (Deb, 2001). Usually, an exterior penalty term, which penalize infeasible solutions, is preferred. Based on the constraint violation $\mathrm{g}_{\mathrm{j}}(\mathrm{x})$ or $h_{\mathrm{k}}(\mathrm{x})$ (inequality and equality constraints), a bracket-operator penalty term is added to the objective function and a penalized function is formed:

$$
\begin{gathered}
F(x)=f(x)+\sum_{j=1}^{J} R_{j}\left\langle g_{j}(x)\right\rangle+\sum_{k=1}^{K} r_{k}\left|h_{k}(x)\right|, \\
g_{j}(x) \leq 0, j=1, \ldots, s \\
h_{k}(x)=0, \quad j=s+1, \ldots m
\end{gathered}
$$

Where $f(x)$ is the objective function, $s$ is the number of inequality constraints, (m-s) is the number of equality constraints, and $R_{j}$ and $r_{k}$ are user-defined penalty parameters. The bracketoperator $<>$ denotes that absolute value of the operand, if the operand is negative. Otherwise, if the operand is non-negative, it returns a value of zero. A recent study suggested a modification by (Deb and Goel, 2000), which eliminates both the above difficulties by not requiring any penalty parameter: 


$$
\begin{aligned}
& \text { If } \mathrm{x} \text { is feasible; } \\
& F(x)=f(x), \\
& \text { Else } \\
& F(x)=f_{\max }+\sum_{j=1}^{J}\left\langle g_{j}(x)\right\rangle+\sum_{k=1}^{K}\left|h_{k}(x)\right|
\end{aligned}
$$

Here, $f_{\max }$ is the objective function value of the worse feasible solution in the population.

The general flow chart for the optimisation process using the constraint DE for the battery network is presented below and illustrated in Fig. 3. The optimisation process can be performed via an automatic process. In order to do so, the user may need to write two small programs for the process. The first program is used to change the parameters of input files of the network model and the second program is used to calculate and evaluate the objective function value. As the standard search progresses, the entire population tends to converge towards the global optimum. This process is continued until a satisfied condition is met. The termination criterion for the iterations is determined according to whether the max iteration or a designed value of the objective function is reached. 


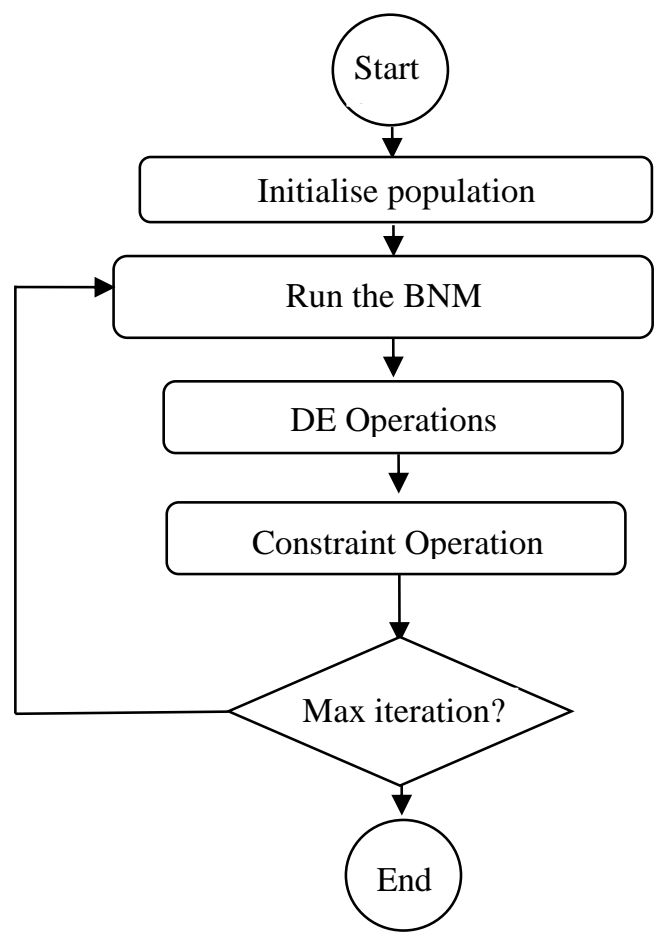

Figure 3: Outline of constraint DE for the battery network

\section{Experimental Setup and Results}

In this section we discuss the experimental setup and simulated results with our storage control strategy. The SACM was introduced to reduce peak demand and this technique has shown great promise in real applications (Rowe et al., 2012). In this study, we investigate the performance of SACM and our proposed BNM with optimal management strategy on New York City, USA. The main objective is to examine whether the BNM would outperform better than SACM. Additionally, the experiment would also reveal whether the BNM would have any particular difficulties or preferences. In order to check the battery network model efficiency and visualize the model performance easily, three batteries have been applied for the case study. The system is composed of the following: wind power generation system, gas power generation system, three batteries and three energy supply areas, as Figure 4 . The parameters $w_{n j}$ of the battery network 
models cannot, in general, be determined directly from mathematical analysis, and hence the parameter values must be estimated by optimisation. In order to minimize the function $P_{r}$, it is necessary to minimize the function $B M$. In our research we used the constraint DE developed in Matlab. The lower and upper bounds weight parameters are equal to $\left[-B_{c},+B_{c}\right]$. The total number of parameters is equal to $20 \times 3=60$ using $\mathrm{DE}$ optimisation when we simulate 20 hours period using BNM. The relevant experimental parameters using the battery network with DE optimisation are listed in Table 1 and Table 2. For the battery network optimisation, an iteration limit of 3000 was employed as a stopping criterion for DE when a population size of 100 was used. The computational time of DE optimisation was about 1 hour on an Intel core 2 Quad CPU 3.40GHz and 8GB of RAM.

We report the results obtained from performing 10 random runs of the battery network algorithm. Figure 5 shows the iteration versus objective function value produced by DE. The DE converged towards to global optima after we ran the constraint DE for about 100 iterations. The left plots of Figure 6 show three actual normalized demand data randomly extracted from New York City demand data. In order to demonstrate the algorithm, 20 hours sampled data were used for the experiment. From Tables 3, it is seen that the battery network model shows a much better result. The small standard deviations (STD) of the performance by the method BNM using DE optimisation imply that the model is stable. Thus, it is clear that the battery network model with DE optimal control considered here is capable of removing more peak areas efficiently than the self-adaptive control model for an individual battery. The above results indicate the average percent of peak areas using BNM is around 20.91 percent more than the SACM. This implies that we get a considerable advantage by using the BNM. This outcome may be easily explained. We assume three batteries are empty for analysis at beginning of simulation. During early hours, 
the battery (1) is fully charged since its actual demand is very low. However, the battery [3] and [2] need extra electricity supply to remove their peaks. When the BNM is optimised, the constraint DE will give optimal weights of the whole simulated period for sharing the energy among the three batteries. This process can balance batteries energy sharing during $\Delta t_{l}$. Hence, performance studies in Table 3 and Figure 6 (b), (d) and (f) reflect the optimal management removing more peaks and head towards to the global optimum. The dot line denotes the battery charge ability and requirements to remove the peak demand, star line the self-adaptive control performance, the circle line the battery network performance in Figure 6 (b), (d) and (f). The negative values in these plots indicate the energy requirements to remove the peaks. During the later hours, the BNM will achieve the similar bad performance as SACM. This is because the three actual demands are all high and there is no energy for sharing. Overall, the results obtained also imply that the BNM is capable of removing the more peaks compared to SACM. The performance of BNM depends on forecasted demand data used for optimal management of batteries and the optimal management algorithm. The robust network modeling technique for energy sharing and the optimal control process are the key reasons for the improvements in cut of more peaks than the SACM.

In the experiment, we assume that demand is perfectly predictable even though there is a small error between forecasted and actual demand data. The uncertainty analysis of input data of forecasted demand also needs to be studied in the future. 
Table 1: Experimental parameters using constraint DE

\begin{tabular}{|c|c|c|}
\hline Parameter & Description & Range \\
\hline$F$ & Control Parameter & 0.5 \\
\hline$C R$ & Crossover Rate & 0.9 \\
\hline$G$ & The total iterations & 3000 \\
\hline$P$ & Population Size & 100 \\
\hline
\end{tabular}

Table 2: Model Parameter Settings

\begin{tabular}{|c|c|c|}
\hline Model parameters & Parameter description & $\begin{array}{c}\text { Suggested values } \\
\text { (after normalize data) }\end{array}$ \\
\hline$B_{c}$ & Capacity of the low battery & $0.1(\mathrm{MWh})$ \\
\hline$T S$ & $\begin{array}{c}\text { Actual supply threshold or } \\
\text { limit for the safety }\end{array}$ & 0.65 \\
\hline
\end{tabular}

Table 3: Comparison of two models

\begin{tabular}{|c|c|c|c|}
\hline Model & $\begin{array}{c}\text { Percentage } \\
(\%) \text { for } \\
\text { battery } 1\end{array}$ & $\begin{array}{c}\text { Percentage } \\
(\%) \text { for } \\
\text { battery } 2\end{array}$ & $\begin{array}{l}\text { Percentage }(\%) \\
\text { for battery } 3\end{array}$ \\
\hline $\begin{array}{l}\text { Three independent batteries using } \\
\text { SACM }\end{array}$ & 30.40 & 18.13 & 7.82 \\
\hline $\begin{array}{l}\text { Average performance of the BNM for } 10 \\
\text { random runs }\end{array}$ & 40.17 & 51.86 & 27.05 \\
\hline STD & 1.2 & 2.6 & 1.6 \\
\hline
\end{tabular}




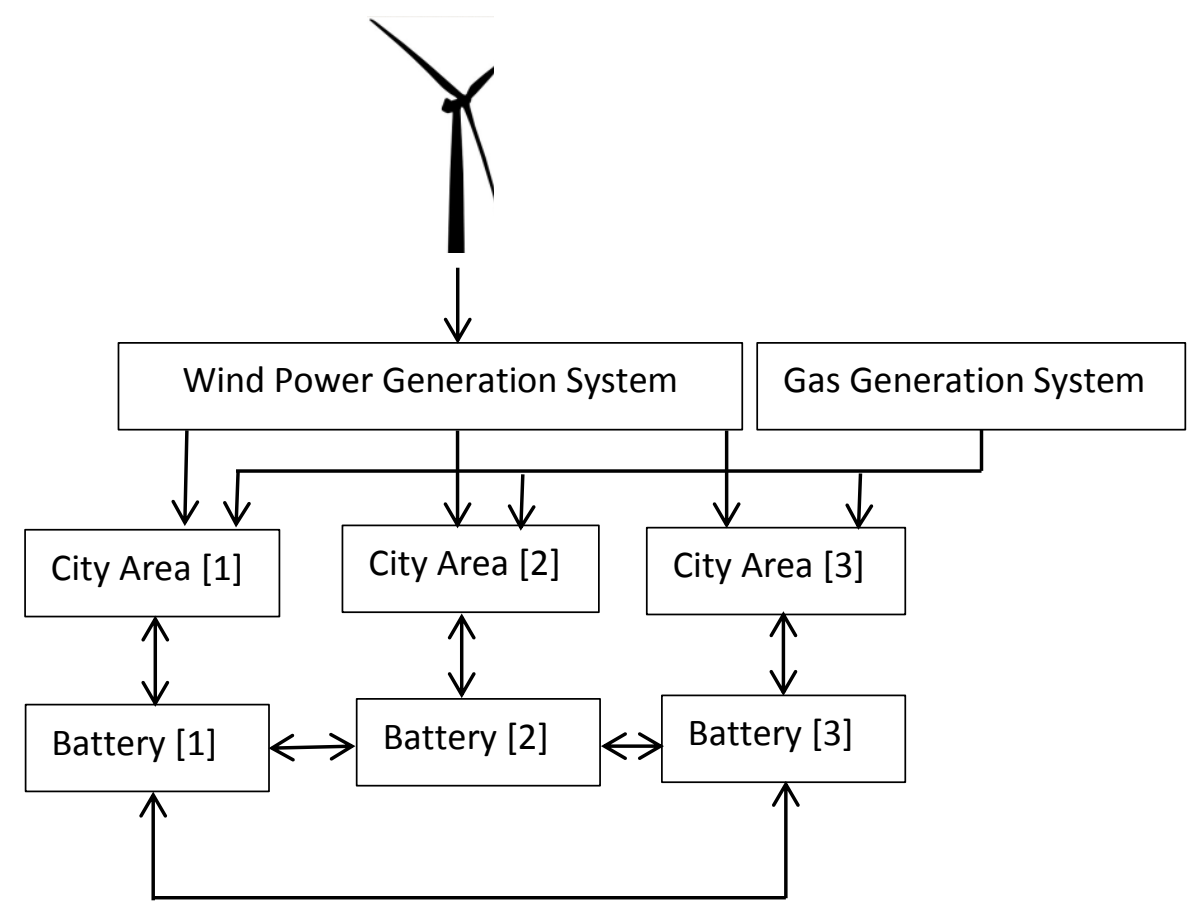

Figure 4: The composition of the energy supply system of New York City

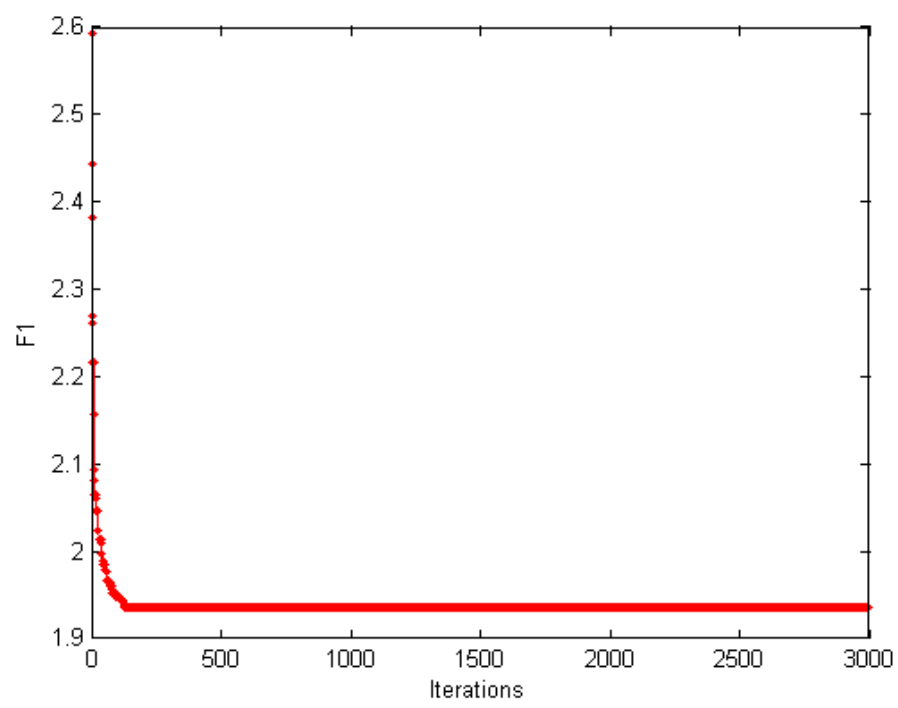

Figure 5: constraint $\mathrm{DE}$ iteration process $(\mathrm{F} 1=B M)$ 


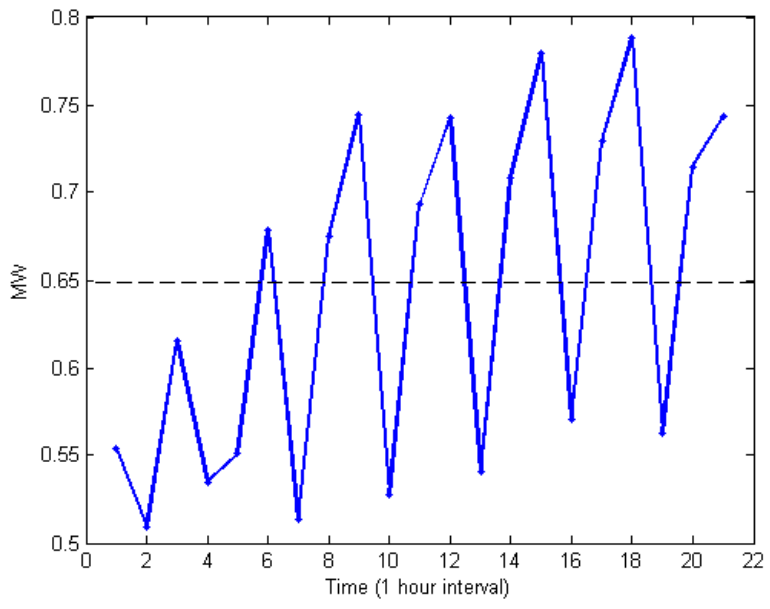

(a)

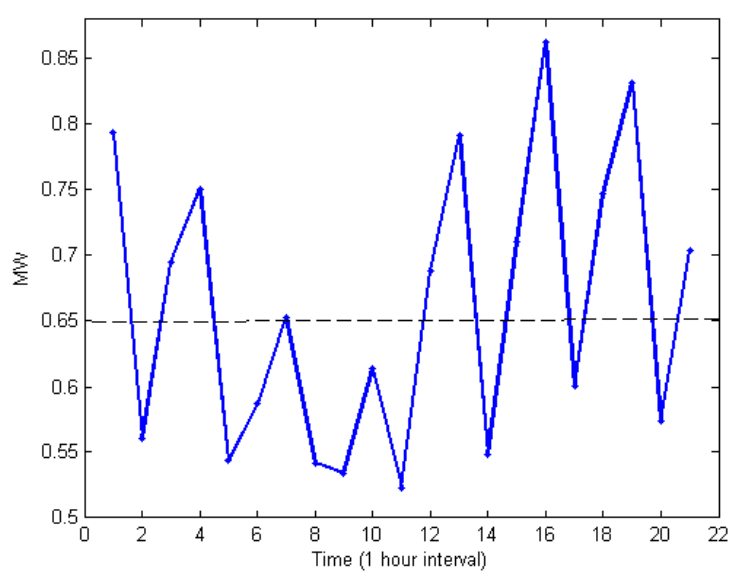

(c)

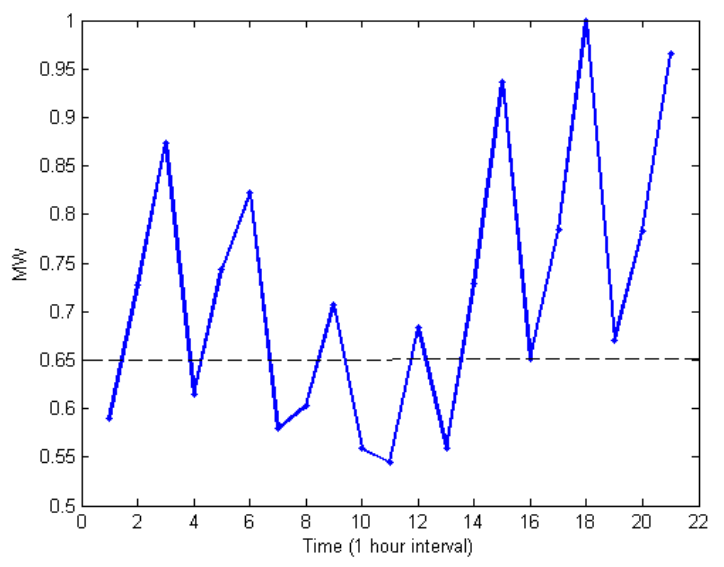

(e)

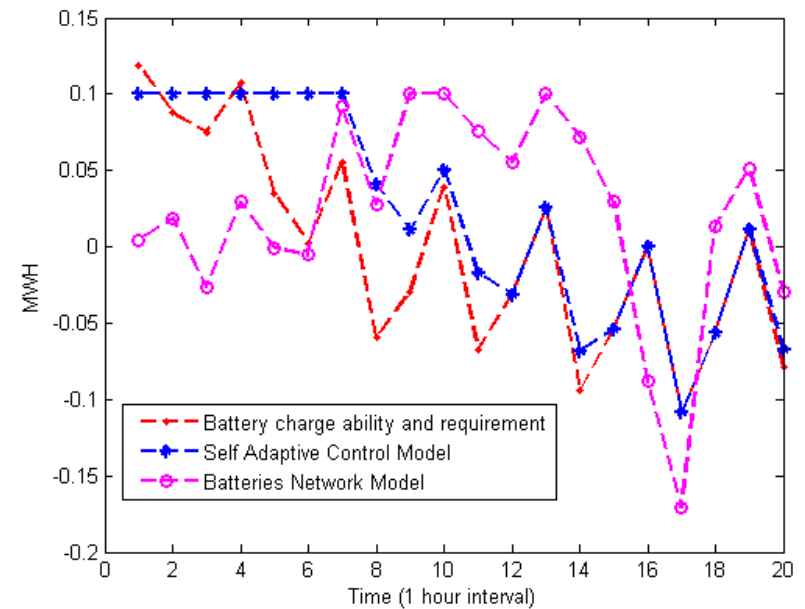

(b)

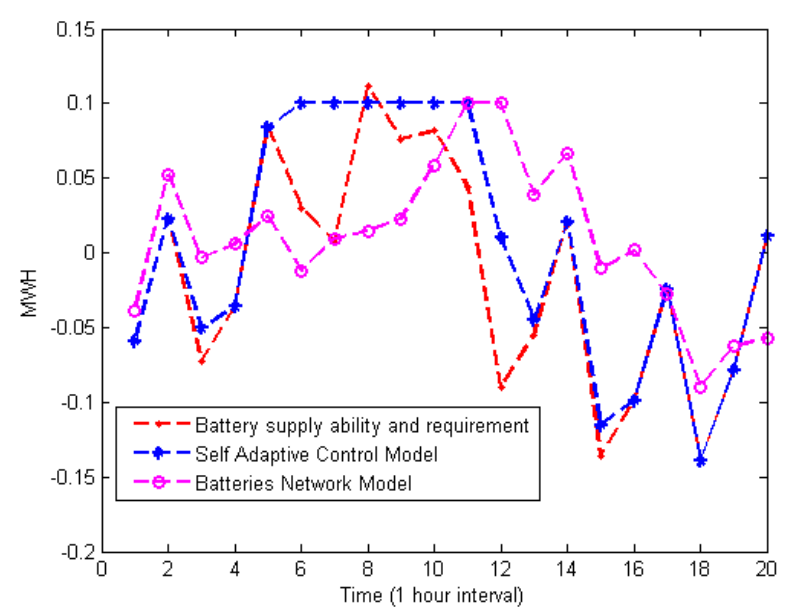

(d)

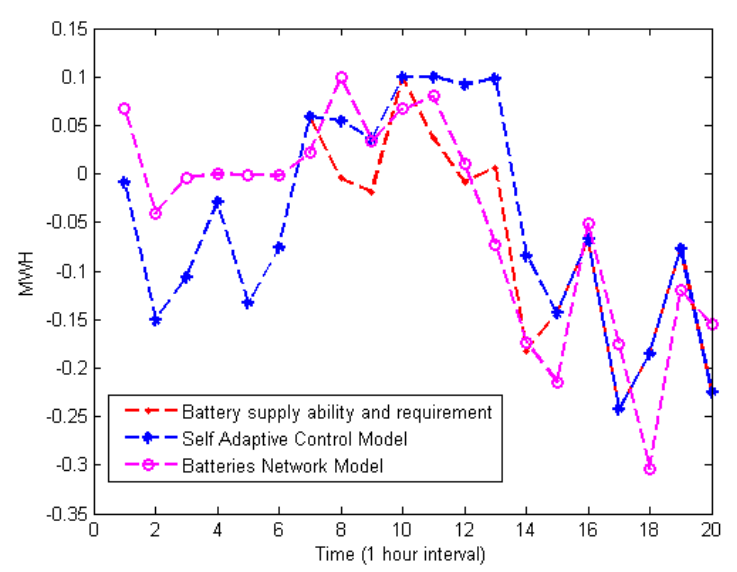

(f)

Figure 5: Left plots are three actual demand data with the threshold (TS $=0.65$ for the normalized data); Right plots are battery supply ability and requirements, self-adaptive model and battery network model performance. 


\section{Conclusions:}

The use of battery storage devices has been advocated as one of the main ways of improving the power quality and reliability as well as minimization of energy imbalance of electricity networks. Higher peaks in demand will also increase the electricity price of market and could cause blackouts and infrastructure damage. The proposed novel battery network model using constraint differential evolution algorithm optimisation has been applied to New York City study case. The DE for the optimal management is simple, robust, and converges fast, and finds the good solution in almost every run. In addition, it has few parameters to set and the same settings can be used for many different applications. The test results showed that the average percent of peak demand areas using the proposed novel BNM is around 20.91 percent more than the SACM. The evaluation scheme considers mathematical model performance measures of two different objectives: (1) peak reduction and (2) comparison to an advanced smart control algorithm (SACM). Work is currently undergoing to include (i) large scale battery network applications, and (ii) uncertainty of input data of demand data.

\section{References:}

Andersson J. (2000) A survey of multi-objective optimisation in engineering design, Technical Report No. LiTH-IKP-R-1097, Department of Mechanical Engineering, Linköping University.

Bejan, A.Iu., Gibbens, R.J., Kelly, F.P. (2012) Statistical Aspects of Storage Systems Modelling in Energy Networks, the 46th Annual Conference on Information Sciences and Systems (invited session on Optimization of Communication Networks), Princeton University, USA. 
Borowy B.S, Salameh Z.M. (1996) Methodology for optimally sizing the combinationof a battery bank and PV array in a wind/PV hybrid system. IEEE Trans Energy Convers, 11(2):36773.

Cai Z., Wang Y. (2006) A multiobjective optimization-based evolutionary algorithm for constrained optimization, IEEE Trans. Evol. Comput.,10 ( 6), 658-675.

Celli G., Mocci S. , Pilo F. and Loddo M. (2009) Optimal Integration of Energy Storage in Distribution Networks, 2009 IEEE Bucharest Power Tech Conference, Bucharest, Romania.

Coello Coello C.A. (2002) Theoretical and numerical constraint-handling techniques used with evolutionary algorithms: A survey of the state of the art, Comput. Methods Appl. Mech. Eng., 191 (11/12), 1245-1287.

Coppez G., Chowdhury S., Chowdhury S. P. (2010) Review of battery storage optimisation in Distributed Generation, in Power Electronics, Drives and Energy Systems (PEDES) \& 2010 Power India, 2010 Joint International Conference on, pp. 1-6.

Cvijovic, D., Klinowski J. (1995) Taboo Search: an approach to the multiple-minima problem, Science, 267: 664-666.

Daly D. (1995) 20mw battery power conditioning system for puerto rico electric power authority. in: Proceedings of the Tenth Annual Battery Conference on Applications and Advances, pp. 233237.

Deb, A., Gupta, B., Roy, J.S. (2010) Performance comparison of Differential Evolution, Genetic Algorithm and Particle Swarm Optimization in impedance matching of aperture coupled microstrip antennas, Mediterranean Microwave Symposium (MMS), 2011 11th, pp.17-20

Deb K. (2001) Genetic algorithms for optimisation. In Technical Report, Kan GAL Report 
No.2001002. Kanpur, India: Department of Mechanical Engineering, IIT Kanpur.

Deb K., Goel T. (2000) Multi-objective evolutionary algorithms for engineering shape design. In Technical Report, Kan GAL Report No.2000003. Kanpur, India: Department of Mechanical Engineering, IIT Kanpur.

Divya K.C., Østergaard J. (2009) Battery energy storage technology for power systems-An overview, Electric Power Systems Research, 79(4), pp 511-520,

Grillo S., Marinelli M., Massucco S., Silvestro F. (2012), Optimal Management Strategy of a Battery-Based Storage System to Improve Renewable Energy Integration in Distribution Networks. IEEE Trans. Smart Grid 3(2): 950-958.

Holland H.J. (2975) Adaptation in Natural and Artificial Systems, An Introductory Analysis with Application to Biology, Control and Artificial Intelligence. Ann Arbor, the University of Michigan Press.

Jin Y. (2005) Comprehensive survey of fitness approximation in evolutionary computation, Soft Computing, 9, 3-12.

Kennedy J., Eberhart R. (1995) Particle Swarm Optimisation. In Proc. of the IEEE Int. Conf. on Neural Networks, pp.1942-1945.

Liu Y. (2009) Automatic Calibration of a Rainfall-Runoff Model Using a Fast and Elitist Multiobjective Particle Swarm Algorithm, Expert Systems with Applications, 36(5): 9533-9538.

Liu Y., Khu S.T. (2007) Automatic Calibration of Numerical Models Using Fast Optimisation by Fitness Approximation, 2007 International Joint Conference on Neural Networks $(I J C N N)(2007)$, pp.1073-1078. 
Liu Y., Pender G. (2012) Automatic calibration of a rapid flood spreading model using multiobjective optimisations, Soft Computing - A Fusion of Foundations, Methodologies and Applications (23 October 2012), pp. 1-12, doi:10.1007/s00500-012-0944-Z.

Liu Y., Pender G. (2013) Carlisle 2005 urban flood event simulation using cellular automatabased rapid flood spreading model, Soft Computing, 17(1), pp. 29-37.

Liu Y. \& Sun F. (2011) A Fast Differential Evolution Algorithm using k-Nearest Neighbour Predictor, Expert Systems with Applications, 38(4): 4254-4258.

Liu Y., Sun F. (2010) Sensitivity analysis and automatic calibration of a rainfall-runoff model using multi-objectives, Ecological Informatics, 5 (4), 304-310.

Mercier P., Cherkaoui R., Oudalov A. (2009) Optimizing a battery energy storage system for frequency control application in an isolated power system, IEEE Trans. Power Syst., 24(3): 1469-1477.

Nguyen C. P. and Flueck A. J. (2012) Agent based restoration with distributed energy storage support in smart grids, IEEE Trans. on Smart Grid, 3(2): 1029-1038.

Nourai A. and Schafer C. (2009) Changing the electricity game, IEEE Power Energy Mag., 17 (4):42-47.

Ould Bilal B., Sambou V., Ndiaye P.A., Kebe C.M.F., Nodongo M. (2010) Optimal design of a hybrid solar wind-battery system using the minimization of the annualized cost system and the minimization of the loss of power supply probability (LPSP), Renewable Energy, 35(10):23882390.

Press W.H., Teukolsky S.A., Vetterling W.T. and Flannery B.P. (1992) Numerical Recipes in Fortran, Cambridge University Press, Cambridge. 
Rowe M., Holderbaum W., Potter B. (2012) Technical report indicating smart control solutions to mollify peak demands with storage and generation within the home and with smart storage in the network, 2012 Technical report-H5127900/4B, Department of System Engineering, Reading University, UK.

Shen J. (2006) Optimal estimation of parameters for an estuarine eutrophication model, Ecological Modeling, 191: 521-537.

Storn R., Price K. (1999) Differential evolution: a simple and efficient adaptive scheme for global optimisation over continuous spaces, Journal of Global Optimisation, 11, 341-359.

Tahllam R.S., Eckroad S. (1999) Multimode battery energy storage for custom power applications, Proceedings of the IEEE Power Engineering Society Winter Meeting, 2(31), pp. $1147-1150$.

Taylor P. (1996) Update on the puerto rico electric power authority's spinning reserve battery system, in: Proceedings of the Eleventh Annual Battery Conference on Applications and Advances, pp. 249-252.

Tina G, Gagliano S, Raiti S. (2006) Hybrid solar/wind power system probabilistic modeling for long-term performance assessment., Solar Energy, 80(5), 578-88.

Vesterstroem J., Thomsen R. (2004) A comparative study of differential evolution, particle swarm optimization, and evolutionary algorithms on numerical benchmark problems, in Proc. IEEE Congr. Evolutionary Computation, Portland, pp. 1980-1987.

Vytelingum P., Voice, T.D., Ramchurn, S. D., Rogers, A., Jennings N.R. (2010) Agent-Based Micro-Storage Management for the Smart Grid. In, The Ninth International Conference on Autonomous Agents and Multiagent Systems (AAMAS 2010), pp.39-46.

Wang Y. Cai Z.X, (2012), A Dynamic Hybrid Framework for Constrained Evolutionary Optimization, Systems, Man, and Cybernetics, Part B: Cybernetics, IEEE Transactions on, 42(1), 203-217. 
Zhou S.Q., Kang L.Y., Guo G.F., Zhang Y.N., Cao J.B., Cao B.G., (2008) The application of combinatorial optimization by Genetic Algorithm and Neural Network, Industrial Electronics and Applications, pp.227-231.

Zhou W., Lou C.Z., Li Z.S., Lu L. and Yang H.X. (2010) Current status of research on optimum sizing of stand-alone hybrid solar-wind power generation systems, Applied Energy, 87:380-389. 